\title{
A Self-Organizing Cooperation for Autonomous Agents Based on Immune Network
}

\author{
Guohua Zhu ${ }^{1}$, Yi Shen ${ }^{1,2, *}$ Xiaobin Hua ${ }^{1}$, Mingxin Yuan ${ }^{1,2}$ \\ ${ }^{I}$ (School of Mechanics and Automotive Engineering, Jiangsu University of Science and Technology, \\ Zhangjiagang, China) \\ ${ }^{2}$ (Suzhou Institute of Technology, Jiangsu University of Science and Technology, Zhangjiagang, China)
}

\begin{abstract}
In order to solve the cooperation of autonomous agent systems, an artificial immune network cooperation algorithm is proposed, which is based on Jerne's idiotypic immune network hypothesis. A sheepdog herding problem is taken as an example. The relative position information of dogs with the sheep and sheep pen is taken as an antigen. Dogs' action is taken as an antibody. We calculate antibody concentration according to Farmer's kinetic model. By selecting the appropriate antibody according to the antibody concentration the cooperation for autonomous agent systems can be realized. Simulation of sheep-dog herding in different environment shows that a herding strategies based on immune network can not only realize sheep-dog herding but also be more robust and flexible, which shows the effectiveness of the artificial immune network algorithm in autonomous agents systems.
\end{abstract}

Keywords: Immune network, Autonomous agents, Planning and cooperation, Sheep-dog herding

\section{Introduction}

The research of multi-robot system is an important branch of artificial intelligence. Planning and cooperation are the key to autonomous agent systems, which has become research hot and difficult in the field of robots. Yang Yupu, et al. ${ }^{[1]}$ presented the further research direction of multi-agent cooperation technologies from the perspective of multi-agent cooperation model and research methods. Based on the idea of multi-agent systems, Chu Yaoqin, et al. ${ }^{[2]}$ proposed a new cooperative control model for the multi-agent systems, which was successfully applied to soccer robot system. This model improved the overall performance of the multiagent system, but the transformation of system role affected the fluency of cooperation and the realization conditions are quite harsh. Yang Gao, et al. ${ }^{[3]}$ promoted a multi-agent negotiation model for virtual enterprise based on contract net, which solved the problems of task allocation and conflict resolution. However, the contract net protocol is not complete and lack of flexible consultation mechanisms. As a result, it can not give full play to the superiority. Xue Hongtao, et al. ${ }^{[4]}$ proposed multi-agent system structure and cooperative approach based on co-evolutionary mechanism. The model system is simple and can promote cooperation of agents, but evolution is uncertain and is not easy to control. It may sometimes affect cooperative performance. In order to improve the planning and cooperative performance of multi-agent system. Aiming at the sheep-dog herding problem of autonomous agents, an artificial immune network cooperation algorithm is proposed to solve the multi-robot cooperation. The simulation results also verify that the proposed algorithm is effective.

\section{Cooperation of Multi-Agent Systems}

In autonomous agent systems, agents can not only be able to plan their own behaviors, but also they must cooperate with other agents, so that they can maintain orderly system and finish the final goal. Sheep-dog herding is a classic problem in the autonomous agent systems. It can be described like this: a few dogs guide a few sheep in the specified environment. Dogs and sheep can not cross the boundary line. Dogs decide their grazing strategies according to the relative position information of themselves with sheep and sheep pen. The final goal is that dogs prevent sheep from moving away from the sheep pen and send sheep into the sheep pen. One dog and one sheep problem is described in Fig. 1. Considering that the efficiency of sheep-dog herding depends directly on the strategy, the artificial immune network ${ }^{[5-6]}$ is introduced in this paper. The sheep and the grazing behaviors are taken as the antigen and the antibody, respectively. Based on Farmer's kinetic model, the grazing behaviors are selected to complete the sheep-dog herding.

\subsection{Idiotypic immune network hypothesis}

\section{Planning Algorithm Based on Immune Network}

Biological immune system is a complicated system, and can prevent the invading of outside pathogen and clear invading pathogens and other harmful substances. In 1973, based on the understanding to the idiotypic * Corresponding author. E-mail: sheny456@ hotmail.com 
characteristic of antibodies, the famous idiotypic immune network hypothesis is developed by Jerne ${ }^{[7]}$. In the idiotypic immune network hypothesis, the body is in a stable state before the outside material go into the body. When antigens invade the body, the balance is broken. B cells are stimulated to produce a number of antibodies, which can recognize antigens. At the same time, to other B-cells, these antibodies are seen as antigens. As a result, other B cells also produce many antibodies, which can recognize and control foregoing antibodies. Antibodies recognize antigens and are recognized by other antibodies. The Idiotypic network hypothesis can be schematically shown in Fig.2. When antigen Ag invades the body, its idiotype is identified by the paratope of Bcell\#1 and antibodies are produced, which can identify antigens. At the same time, to B-cell\#2 and B-cell\#3, antibodies that are produced by B-cell\#1 are considered as antigens. Therefore, B-cell\#2 and B-cell\#3 will produce a large number of antibodies to identify and control antibodies that are produced by B-cell\#1. Of course, B-cell\#2 and B-cell\#3 will control each other. By the interaction of the antibody with the antigen and the antibody, antigens can be wiped out effectively and excessive antibodies can be controlled. Finally, the balance will be maintained.

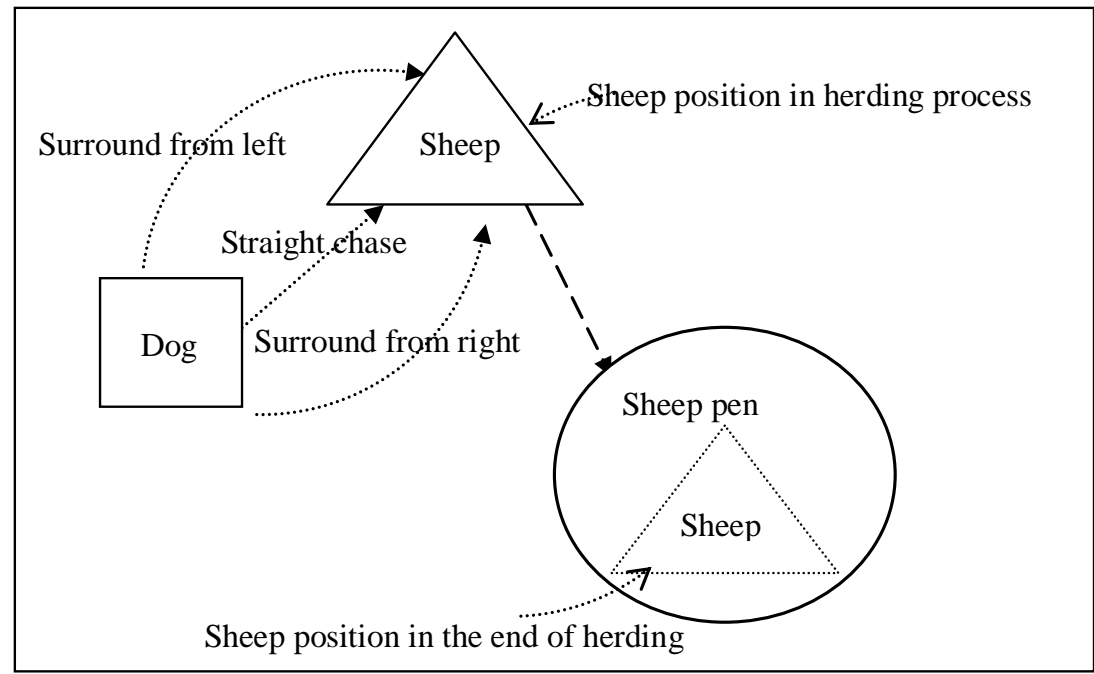

Fig. 1 One dog and one sheep problem

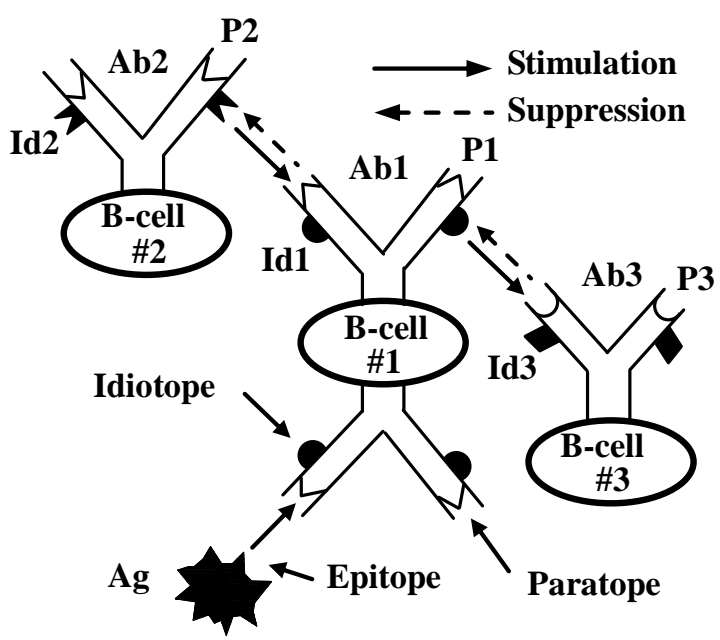

Fig. 2 Idiotypic immune network hypothesis

\subsection{Sheep-dog herding model based on immune network}

To solve the sheep-dog herding problem in autonomous agent systems, an artificial immune network planning and cooperation model based on Jerne's idiotypic immune network hypothesis ${ }^{[8-9]}$ is presented. In this model, the dog is taken as an autonomous agent and the dog's behaviors are taken as antibodies. The dog is equipped with eight non-uniform virtual sensors as shown in Fig. 3. In the planning system, according to the environmental information, three moving commands of a dog are set, namely straight chasing, surrounding 
from left and surrounding from right. When all the sheep are sent into the sheep pen, dogs stop moving and the task is completed.

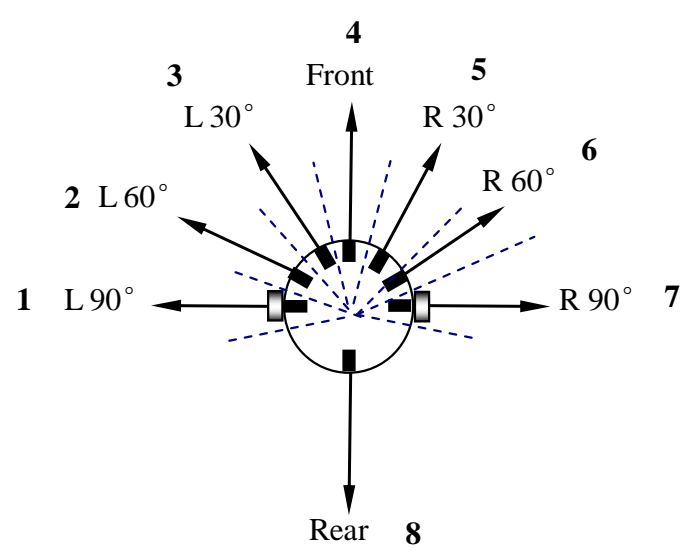

Fig. 3 Dog with eight virtual sensors

Table 1 show the corresponding relationship between the sheep-dog herding problem and immune network.

Table 1 Relationship between the sheep-dog herding problem and immune network

\begin{tabular}{cc}
\hline Sheep-dog herding problem & Immune network \\
\hline Relative position information of dogs with & Antigen \\
sheep and sheep pen & Antibody \\
Grazing strategy & B cell \\
Dog & Stimulation \\
Adaptation & Suppression \\
Non-adaptation &
\end{tabular}

\subsection{Definition of antigen}

\section{Immune Network herding Algorithm}

In the sheep-dog herding problem, the antigen is described as the relative position information of dogs with sheep and sheep pen, which can be obtained by detecting by eight virtual sensors around the dog. Antigen can be achieved by binary coding. To one virtual sensor direction, if there is a sheep or sheep pen on this direction, the direction is marked with 1 . Otherwise, the direction is marked with 0 . For example, if the dog detect a sheep on the 2nd direction and detect the sheep pen on the 4th direction, the antigen can be described like this:

\begin{tabular}{|l|l|l|l|l|l|l|l|l|l|l|l|l|l|l|l|}
\hline 0 & 1 & 0 & 0 & 0 & 0 & 0 & 0 & 0 & 0 & 0 & 1 & 0 & 0 & 0 & 0 \\
\hline
\end{tabular}

The front 8 bits are information of sheep, and the back 8 bits are information of sheep pen.

\subsection{Definition of antibody}

In the sheep-dog herding problem, antibody is described as strategies of dogs during the herding. Antibody is made up of antibody paratope and dog actions. The coding of antibody paratope includes sheep and sheep pen, shown as Table 2 . There are 16 directions. The front 8 directions are coding information of sheep and the back 8 directions are information of sheep pen.

Table 2 Definition of antibody of paratope

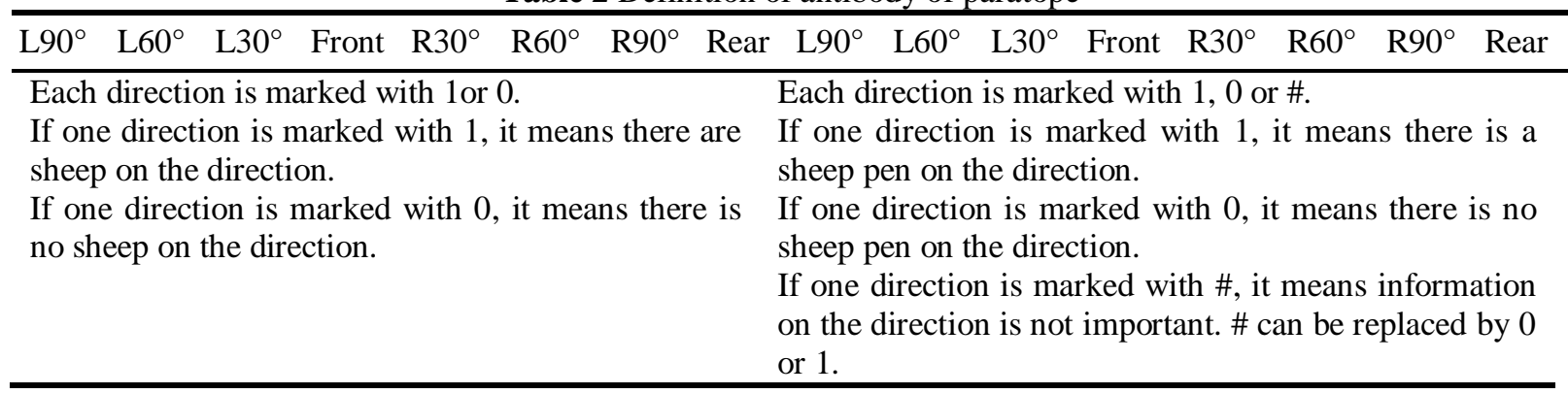


For example, if there is a sheep on the $3^{\text {rd }}$ direction and the sheep pen is on any direction of $4^{\text {th }}, 5^{\text {th }}, 6^{\text {th }}$, $7^{\text {th }}$ and $8^{\text {th }}$ directions, the dog will select the action of surrounding from left. This antibody can be shown as follows:

\begin{tabular}{|l|l|l|l|l|l|l|l|l|l|l|l|l|l|l|l|l|}
\hline 0 & 0 & 1 & 0 & 0 & 0 & 0 & 0 & 0 & 0 & 0 & $\#$ & $\#$ & $\#$ & $\#$ & $\#$ & $\begin{array}{l}\text { Surround } \\
\text { from left }\end{array}$ \\
\hline
\end{tabular}

According to the relative position information of dogs with sheep and sheep pen as well as dogs' strategies, 24 antibodies are defined in this paper.

\subsection{Calculation and selection of antibody concentration}

In order to make the dog select the better strategy during the herding, the antibody concentration is calculated by using Farmer's kinetic model. Then the antibody can be selected reasonably.

Calculation of antibody concentration is shown as follows:

$$
\begin{gathered}
\frac{d a_{i}^{o}(t)}{d t}=\left(\alpha \frac{\sum_{j=1}^{N}\left(m_{j i}^{o} a_{j}^{o}(t-1)\right)}{N}-\alpha \frac{\sum_{j=1}^{N}\left(m_{i j}^{o} a_{j}^{o}(t-1)\right)}{N}+\beta m_{i}^{o}-k_{i}^{o}\right) \times a_{i}^{o}(t-1) \\
S_{i}^{o}(t)=a_{i}^{o}(t-1)+\frac{d a_{i}^{o}(t)}{d t} \\
a_{i}^{o}(t)=\frac{1}{1+\exp \left(0.5-S_{i}^{o}(t)\right)}
\end{gathered}
$$

In Eq.(1), $N$ represents the number of antibodies; $\alpha$ and $\beta$ are positive adjustment coefficient. The first and the second in the bracket of the right side of Eq. (1) successively represent the stimulation and suppression that other antibodies act on the antibody $i . m_{j i}^{o}$ and $m_{i j}^{o}$ represent the stimulating factor and inhibiting factor that antibody $j$ acts on antibody $i$, respectively. $m_{i}^{o}$ represents the stimulating factor that the antigen acts on antibody $i . k_{i}^{o}$ represents the natural aging and death of antibodies.

The $m_{j i}^{o}, m_{i j}^{o}$ and $m_{i}^{o}$ can be calculated as follows:

$$
\begin{aligned}
& m_{j i}^{o}=\left[\sum_{l} p_{j}^{o}(l) \oplus \overline{p_{i}^{o}(l)}\right] / n \\
& m_{i j}^{o}=\left[\sum_{l} p_{j}^{o}(l) \oplus \overline{\left.p_{i}^{o}(l)\right] / n}\right. \\
& m_{i}^{o}=\left[\sum_{l^{\prime}} E^{o}\left(l^{\prime}\right) \oplus \overline{p_{i}^{o}\left(l^{\prime}\right)}\right] / n^{\prime}
\end{aligned}
$$

In above equations, $\oplus$ represents xor operation. $p_{j}^{o}(l)$ and $p_{i}^{o}(l)$ represent the paratope information of antibody $j$ and $i$, respectively. $E^{o}\left(l^{\prime}\right)$ represents the coding information of the antigen.

\subsection{Main steps of the herding algorithm}

Step 1 Initialize the immune network status, including the initial value of antibody concentration and some adjustment coefficients. As well as, initialize position coordinates of dogs, sheep and sheep pen.

Step 2 Calculate the distance between the dog and the sheep. If the distance is greater than a certain value of the algorithm, the dog straight chases the sheep and go to step 6. Otherwise, the dog equipped with eight nonuniform virtual sensors achieves coding information of the antigen and then select antibody number preliminarily according to the information that sensors detect.

Step 3 Calculate stimulating values and inhibiting values among selected antibodies, and the stimulating values that the antigen acts on antibodies.

Step 4 Calculate the concentration of each antibody according to (1), (2), (3). 
Step 5 Select an antibody whose concentration is the largest and then determine the dog's strategy according to the selected antibody.

Step 6 Update position coordinates of dogs and sheep.

Step 7 Judge whether all sheep are in the sheep pen. If not, go to step 2, otherwise the grazing task is finished.

\section{Tests of sheep-dog herding}

In order to further validate the effectiveness and superiority that the immune network planning algorithm in solving sheep-dog herding problem, aiming at the following four cases, some tests are carried out on the computer with PIV 2.10GHz 2.00GB RAM by using Matlab7.0. Some key parameters are set as: $\alpha=0.1$, $\beta=0.5, k_{i}^{o}=0.005$, initialization concentration of antibodies are all 0.1 , the speed of the sheep is $0.9 \mathrm{~m} / \mathrm{s}$ and the speed of the dog is $1.2 \mathrm{~m} / \mathrm{s}$.

(1)One dog and one sheep

In one dog and one sheep problem, according to the relative position information of the dog with the sheep and sheep pen, the dog selects a suitable antibody by calculating the antibody concentration based on the distance and direction among the dog, sheep and sheep pen.

Fig. 4 and Fig. 5 are the test results of the proposed herding algorithm in two different environments. In Fig.4, the starting position of the dog is $(1,10)$. The angle between the positive $\mathrm{x}$-axis and the dog's direction is $0^{\circ}$. The starting position of the sheep is $(16,16)$. In Fig.5, the starting position of the dog is $(19,6)$. The angle between the positive $\mathrm{x}$-axis and the dog's direction is $0^{\circ}$. The starting position of the sheep is $(10,16)$. The sheep pen is a circle whose center is $(10,10)$ and radius is 2 .

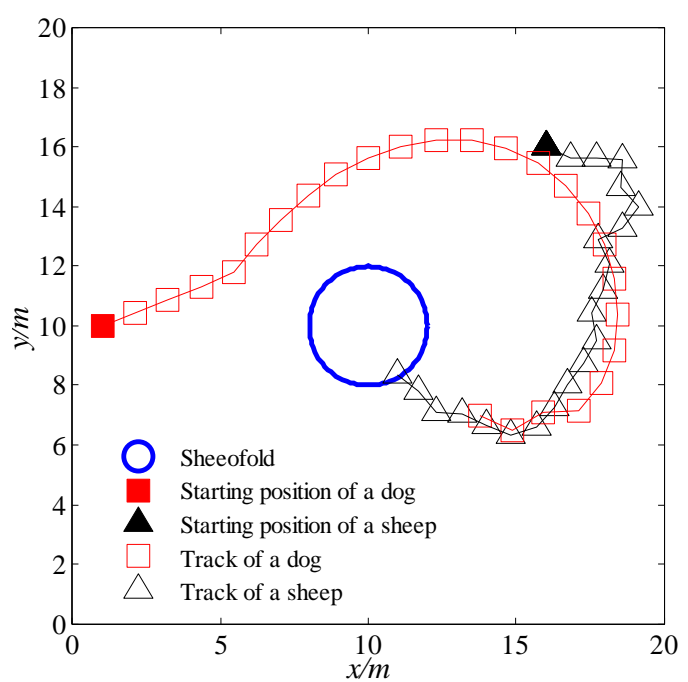

Fig. 4 Test one of one dog and one sheep

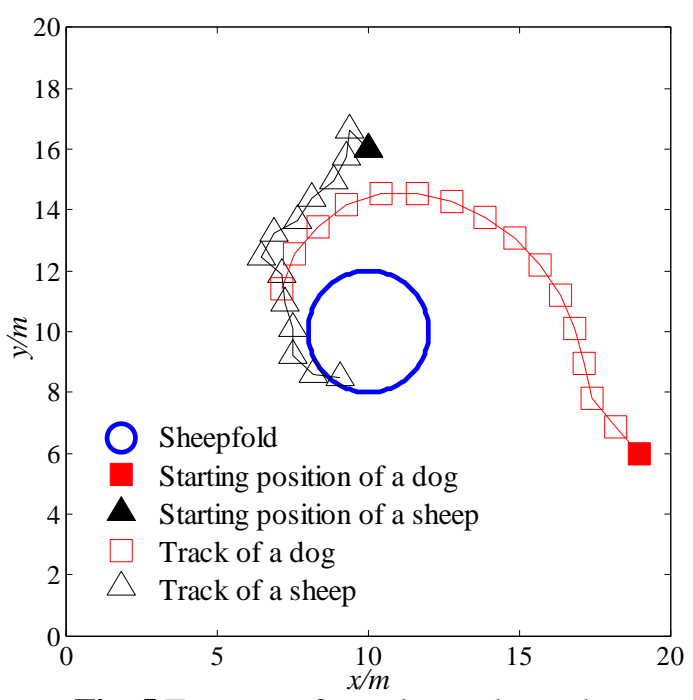

Fig. 5 Test two of one dog and one sheep

Fig.4 shows that at the beginning, the dog is relatively far away from the sheep. The dog straight chases the sheep and the sheep is unaware of the danger without moving. As the dog gradually gets close to the sheep, the sheep begins to escape. The final antibody is selected through preliminary selection of antibodies and calculation of antibody concentration. The dog adopts the appropriate strategy according to the selected antibody. As shown in Fig.4, in the later stage, the dog takes the action of surrounding from left. Finally, the task is completed. Fig.5 is the simulation result in another environment. By calculating antibody concentration and selecting antibodies, the dog straight chases the sheep at the first and then surrounds the sheep from right. In the end, the sheep is in the sheep pen.

\section{(2) One dog and two sheep}

In one dog and two sheep problem, the dog needs to plan its own behaviors. According to the relative position of the dog with two sheep and sheep pen, as well as the relationship of distances between two sheep and the sheep pen, the dog determines the order of chasing sheep and selects the suitable strategy. In the end, the two sheep are driven into the sheep pen.

Fig. 6 and Fig.7 are the test results of the proposed algorithm in two different environments. In Fig.6, the starting position of the dog is $(1,10)$. The angle between the positive $\mathrm{x}$-axis and the dog's direction is $90^{\circ}$. The starting positions of the two sheep are $(10,16)$ and $(18,4)$; In Fig.7, the starting position of the dog is $(16$, 1 ). The angle between the positive $\mathrm{x}$-axis and the dog's direction is $0^{\circ}$. The starting positions of the two sheep are $(4,12)$ and $(16,16)$. The sheep pen is a circle whose center is $(10,10)$ and radius is 2. 


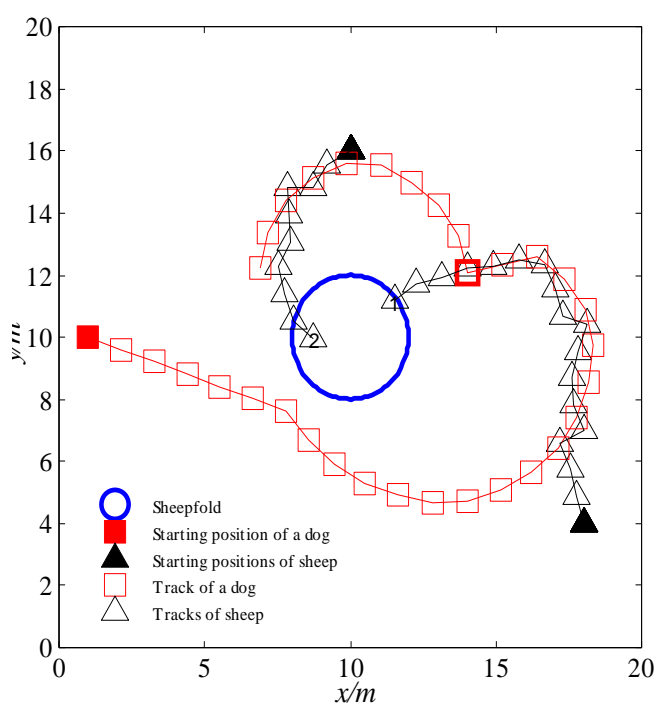

Fig. 6 Test one of one dog and two sheep

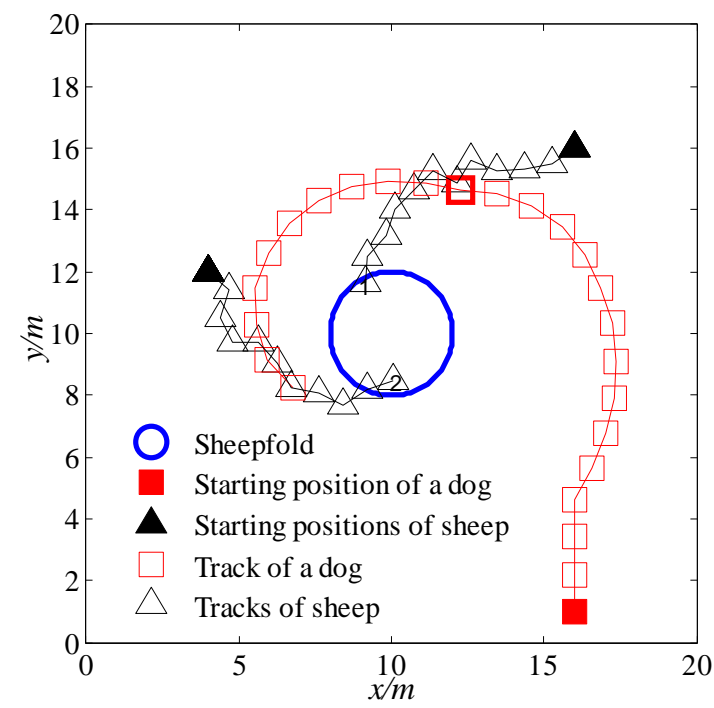

Fig. 7 Test two of one dog and two sheep

In Fig.6, first, the dog chases sheep1 that is farther away from the sheep pen. At the beginning, the dog caches sheep1 because sheep1 is far away from the dog. Then, according to the detection of positions, calculation of antibody concentration and selection of antibodies, the dog surrounds sheep1 from right and sheep1 is driven into the sheep pen in the end. After that, the dog begins to chase sheep2. As shown in Fig.7, the dog surrounds sheep 2 from right. Finally, the two sheep are driven into the sheep pen and the task is completed. Fig.7 is the simulation result in another environment. According to detection of positions, calculation of antibody concentration and selection of antibodies, the dog first chases sheep1 and then chases sheep2. Finally, the two sheep are also in the sheep pen.

\section{Conclusions}

In order to solve the sheep-dog herding problem, the planning and cooperation of autonomous agents systems is discussed and studied. Finally, an artificial immune network cooperation algorithm is proposed. The characteristic of the immune network algorithm for sheep-dog herding are as follows:

(1) The relative position information of dogs with sheep and the sheep pen is taken as the antigen.

(2) Dogs' action is taken as the antibody.

(3) The antibody concentration is calculated according to Farmer's kinetic model.

(4) The dogs' strategy is determined according to the antibody concentration.

Simulating results show that the proposed algorithm can solve sheep-dog herding problem in different environments.

\section{Acknowledgements}

This work was supported in part by the National Nature Science Foundation of China (NSFC) under Grant 61105071, Qing Lan Project of Jiangsu Province under Grant Jiangsu teacher [2012]39, industryacademia cooperation innovation fund projects of jiangsu province under Grant SBY201220397.

\section{References}

[1] Y.P. Yang, X.M. Li and X.M. Xu, A survey of technology of multi-agent cooperation, Information and Control, 4, 2001, 337-342.

[2] Y.Q. Chu, X.A. Li and Y. Pu, Cooperative control of multi-agent soccer robot system, Journal of Harbin Institute of Technology, 7, 2004, 911-913.

[3] Y. Gao, W. Zhou and X.Q. Zeng, Multi-agent learning negotiation research in virtual enterprise based on contract net, Computer Integrated Manufacturing Systems, 4, 2004, 471-475.

[4] H.T. Xue, L.C. Shen and Y.Y. Ye, Multi-agent collaboration system architecture and simulation framework based on co-evolution method, Journal of System Simulation, 3, 2002, 297-299.

[5] J. Zhuang, S.A. Wang, Further study of robot path planning algorithm based on artificial immune net theory, Journal of System Simulation, 5, 2004,1017-1019.

[6] S.A. Wang and J. zhuang, The algorithm of the path finding and optimizing of the moving robot, Journal of System Simulation, 8, 2002, 995-998.

[7] N.K. Jerne, Towards a network theory of the immune system, Annual Immunology, 125, 1974, 373-389.

[8] N.K. Jerne, The immune system, Scientific Ametican, 229,1973, 52-60.

[9] N.K. Jerne, Idiotopic network and other preconceived ideas, Immunological Rev, 79, 1984, 5-24.

[10] J.D.Farmer, N.H.Packard and A.S.Perelson, The immune system, adaptation and machine learning, Physical D, $22,1986,187-204$. 